\title{
Hypnotizability and the position sense: proprioceptive localization of the hand
}

\author{
M.A. PADILLA CASTANEDA ${ }^{1,2}$, E. CASTELLANI ${ }^{3}$, A. FRISOLI', M. BERGAMASCO', \\ E.L. SANTARCANGELO ${ }^{1,3}$ \\ ${ }^{7}$ Laboratory of Perceptual Robotics, Scuola Superiore S. Anna, Pisa, Italy; ${ }^{2}$ Centro de Ciencias \\ Aplicadas y Desarrollo Tecnológico, Universidad Nacional Autónoma de México, México City, \\ Mexico; ${ }^{3}$ Department of Translational Research and New Technologies in Medicine and Surgery, \\ University of Pisa, Pisa, Italy.
}

\section{A B S T R A C T}

We investigated whether healthy subjects with high (highs) and low (lows) hypnotizability scores differ in the ability to report the position of their right hand in the horizontal plane at the end of passive and active arm movements directed to lateral, intermediate and medial targets of the right hemispace under correct or incorrect visual feedback. Results showed that incorrect visual feedback increased the error in both groups. In lows, the error was similar after active and passive movements; in highs, it was lower for active than passive movements toward the medial position, but lower for passive than for active movements for the lateral one. The highs' error was significantly lower than the lows' one only for the active movements directed toward the medial hand position. Hypnotizabilityrelated differences may be due to different role of efferent copies in highs and lows.

\section{Key words \\ position sense $\bullet$ Hypnotisability $\bullet$ Virtual reality $\bullet$ Efferent copies}

\section{Introduction}

Hypnotizability is an individual trait associated with the proneness to accept suggestions (Green et al., 2005) and with a number of physiological differences (Huber 2013; Santarcangelo 2014) observed in the ordinary state of consciousness and in the absence of specific suggestions. In particular, hypnotizability accounts for part of the physiological variability of the general population in sensorimotor integration and in sensory imagery (Carli et al., 2008; Santarcangelo 2014; Menzocchi et al., 2015).

Kinestesia seems to have a different relevance in the sensori-motor integration of the subjects with high (highs) and low (lows) hypnotizability in that highs form mental images on the basis of haptic information better than lows. In fact, the visual recognition of haptically explored non meaningful objects is more likely (Castellani et al., 2011) and the blindfolded reproduction of haptically explored angles is more accurate in highs than in lows. Also, kinesthetic imagery is more effective in highs, as they have greater abilities to perceive heaviness and behave accordingly (Santarcangelo et al., 2005) and exhibit greater proneness to choose the proprioceptive modality when they are invited to generate body images contrasting with the real body posture (Santarcangelo et al., 2010) as well as to maintain sensations from this modality when they are asked to suppress all imagined perception (Carli et al., 2007a). Finally, highs report lower effort and higher efficacy in the generation of mental images through the kinesthetic/tactile modality of imagery with respect to lows (Carli et al., 2007b). In brief, findings indicate that the proprioceptive physical 
and imagined sensory modality plays a major role in the highs sensori-motor integration, which suggests an advantage of highs in the position sense. Nonetheless, since the highs's attention has a great proneness to be captured by visual images (Tellegen and Atkinson, 1974), incongruent visual information may impair their kinestetic performance. Both the position sense and the possible competition between the visual and kinaesthetic modality have not been studied.

The position sense can be studied by analyzing the individual ability to detect the position of arms/ hands at the end of passive or active movements. Theoretically, the ability to determine the hand position should be greater when it is reached after active rather than after passive movements owing to the additional information deriving from the efferent copies of motor commands which increase the sensitivity of Ia-spindle afferents by activating $\gamma$-dynamic motoneurones (Adamovich et al., 1998; Craske and Crawshaw, 1975; Fuentes and Bastian, 2010; Gritsenko et al., 2007; Laufer et al., 2001; Medina et al., 2010) and allow to forecast the upcoming location of the limb (Cui et al., 2014; Leube et al., 2003). Nonetheless, recent findings on pointing movements indicate that the so-called active proprioception does not provide better estimates of limb position than the passive one (Capaday et al., 2013).

The aim of the present study was to assess whether highs and lows display different ability to detect the position of their right hand after active and passive movements in the horizontal plane, and to study whether an incorrect visual feedback of the performed movement may impair their performance differentially.

\section{Methods}

\section{Subjects}

After the approval of the experimental procedure by the Ethics Committee of the University of Pisa, 95 unpaid volunteers were recruited among students. They signed an informed consent describing the procedure, but not the aims of the study, and were submitted to hypnotic assessment through the Italian version (De Pascalis et al., 2000) of the Stanford Hypnotic Susceptibility Scale, form C (SHSS). Twenty-two right-handed females (Edinburgh
Handedness Inventory, scores $>14$ in all subjects) were enrolled in the study. They were 11 subjects with high scores of hypnotisability (highs, SHSS score (mean $\pm \mathrm{SD})$ : $10.21 \pm 0.8$, age, $23.2 \pm 1.2)$ and 11 with low scores (lows, SHSS score: 1.05 \pm 0.03 , age, 22.41 \pm 1.6). Highs and lows did not differ significantly in the arm length from the acromion to the

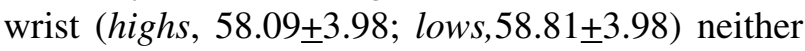
in the length of the upper arm (highs, 30.54 \pm 3.07 ;

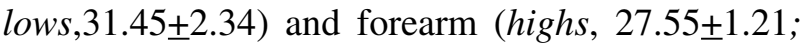
lows,28.36+1.96).

\section{Task}

While holding the end-effector of a robotic arm with their right hand (Bergamasco et al., 2006), participants were instructed to complete horizontal movements towards different positions and to verbally report the perceived position of their hand with respect to a visual reference appearing on a screen at the end of the movement (Fig. 1). With the aim of minimizing the wrist and elbow rotations, the robot constrained the movements of the right hand by exerting repulsive haptic forces when the subject tried to deviate from the radial axial of the workspace. In addition, a rigid bandage was applied on the wrist. As a consequence, the kinesthetic information responsible for detection of the hand final position was predominantly due to the shoulder joint and muscles.

Movements towards three targets -lateral (P1) intermediate (P2) and medial (P3) positions - were performed actively, with the subjects holding and moving the handle (end-effector) of a robotic device, and passively, with the robot placing the subject's hand (Fig. 1).

In the active movement condition, at a beep occurrence participants had to move the robotic handle from its initial zero position, to pay attention to the occurrence of a sound delivered in correspondence of $\mathrm{P} 1, \mathrm{P} 2$ or $\mathrm{P} 3$, which approximately corresponded to one $(15 \mathrm{~cm})$, two $(30 \mathrm{~cm})$ and three thirds of the maximal excursion (mean+SD; $45+2.3 \mathrm{~cm}$ ) achievable through rotation of the shoulder (see Supplementary Electronic Material)-, and interrupt the movement immediately after it. In the active movements subjects placed the robot end-effector at the trained velocity (Supplementary Electronic Material), while in the passive movements the robot placed the subject's hand in space through an imple- 
(a)

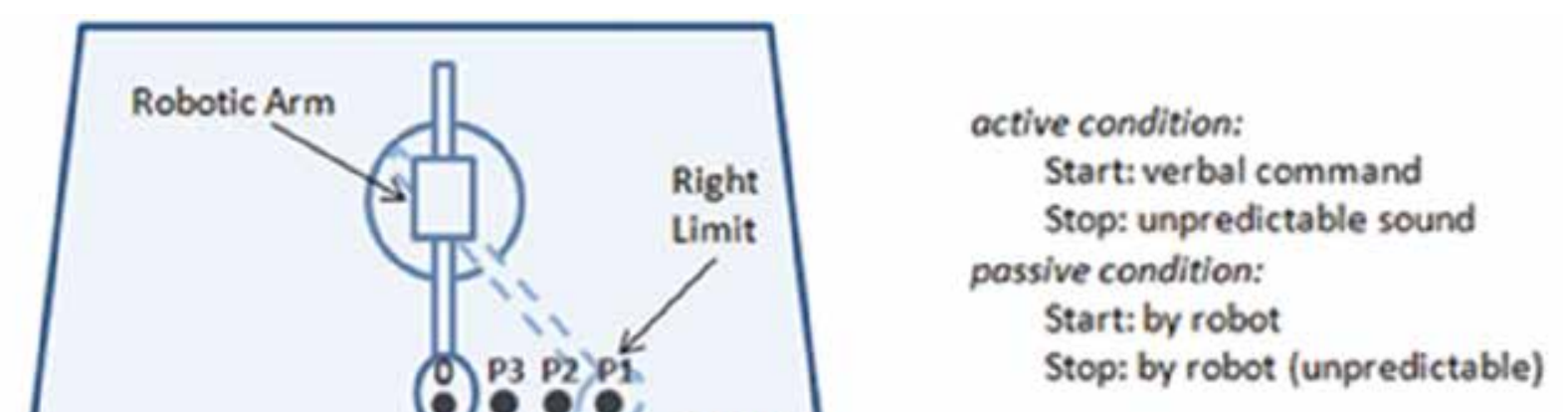

(b)
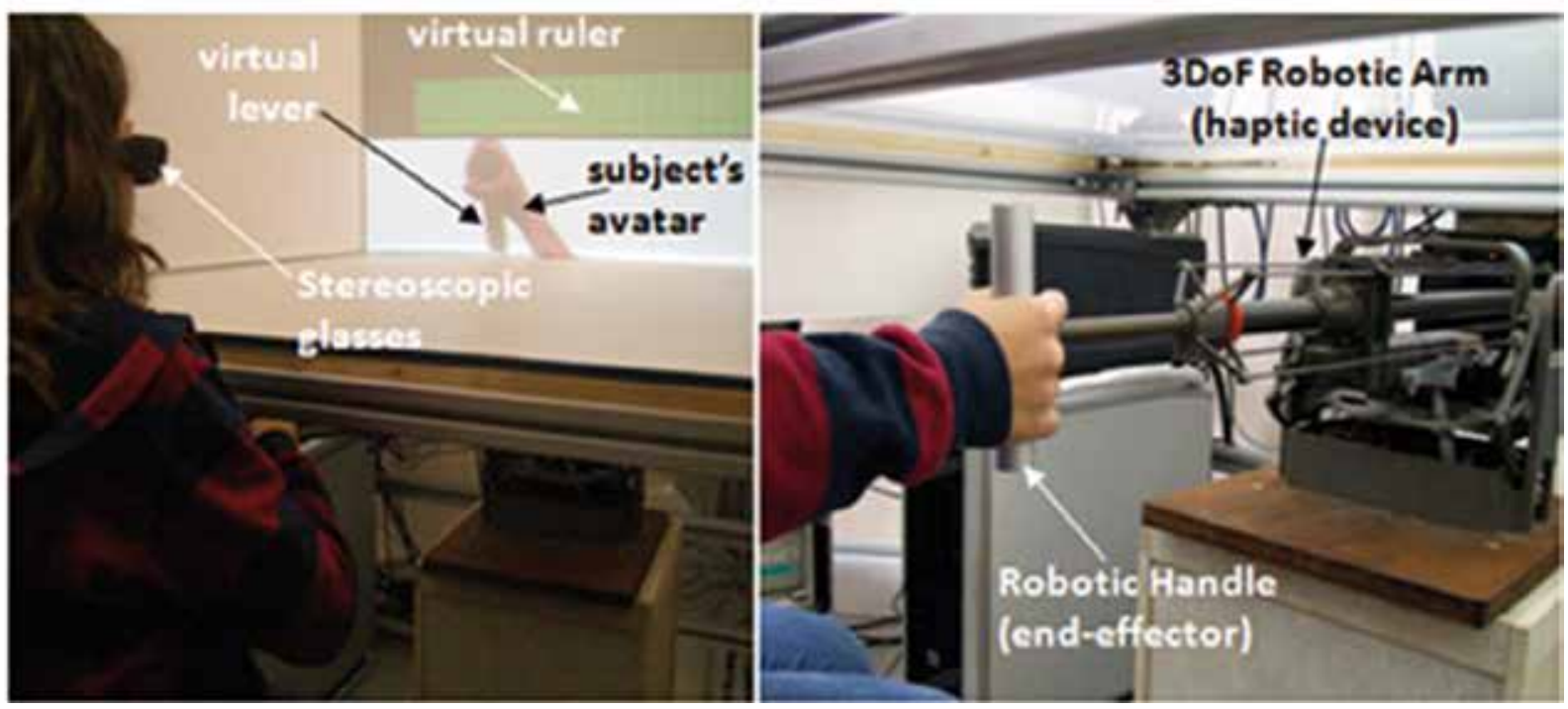

Fig. 1. - Experimental set-up and procedure. A) Schema of the subject movements while holding the robotic arm. Both the participant's and robotic arm were hidden to participants. B) A participant interacting with the experimental setup.

mented control algorithm at the same mean training reference velocity $(13.5 \mathrm{~cm} / \mathrm{s})$ adopted for the active movement (Fig. 1). The excursion of the subjects' joint angles corresponded to $7.55 \pm 4.665^{\circ}$ for the shoulder adduction/abduction, to $24.53 \pm 8.028^{\circ}$ for the shoulder flexion/extension, to $17.10 \pm 11.547^{\circ}$ for the shoulder external rotation and to $77.85 \pm 12.07$ o for the elbow's flexion/extension. There was no hypnotizability-related difference.

Participants received visual feedback by the replication of their movements in real-time with a threedimensional (3D) virtual reality (VR) avatar that simulated the subject's upper limb, projected into an immersive virtual environment (VR Cave) (Padilla-
Castañeda et al., 2014). For half of the trials, a visual feedback not corresponding (incorrect feedback) to the participants real movement was introduced by showing the replicated movement with the avatar drifted towards the direction of the movement (see Supplementary Electronic Material). Participants knew that the visual feedback may correspond or not correspond to their real movement.

\section{Experimental procedure}

Experimental sessions were scheduled at least one month after hypnotic assessment and were performed in the afternoon ( 3 p.m- 6 p.m.) in a dimly light and sound attenuated room. Participants were 
informed about the sequence of tasks, dressed the stereovision glasses, sat in front of the screen looking at the VR upper limb avatar (in a position that provided the same perspective as looking its own right arm), and hold the end-effector of the robot (which was hidden all the time under a cardboard piece) together with the forearm with their right hand. The motions of their right wrist and elbow were minimized given the stiffness of the endeffector without orientations and the haptic forces exerted by the robot within the linear workspace along the $\mathrm{X}$ axis. Minimization of the wrist and elbow movements was necessary to be able to perform the horizontal movement within the range defined in each participant at the beginning of the session as the maximum range allowed by the arm length (see Supplementary Electronic Material). Individual calibration procedures are reported in the Supplementary Electronic Material.

At the beginning of each task, a virtual ruler appeared on the screen and the subject was invited to horizontally adjust the real hand location at the starting position which corresponded to the virtual hand centered at the zero of the virtual ruler. With the real hand placed at zero, the virtual ruler disappeared and the subject was instructed to perform the movement without stopping until hearing a beep sound signal. Immediately after the beep, participants had to stop their movement, the virtual ruler reappeared, the avatar disappeared, and the subject was asked to verbally report in the ruler the perceived location of their real hand. The error of the report corresponded to the absolute values of the difference between the reported and the real hand position (absolute error). Relative values of this difference indicated under/overestimation of the hand final position (constant error). Next, the ruler disappeared once again and with the screen in black the subject was asked to back the real hand position to her perceived zero position. Finally, the avatar and the ruler were shown again, the subject adjusted the hand to the zero and iteratively continued with the next task.

All subjects performed randomly series of tasks (see Supplementary Electronic Material), covering combinations of rightward movements with correct and incorrect visual feedback (correct, incorrect) toward a lateral $(\mathrm{P} 1$, mean distance from zero $(\mathrm{cm})$ :

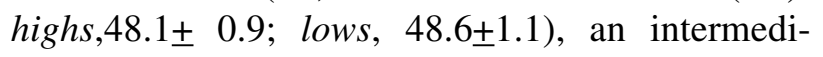

ate (P2, highs, 28.5 \pm 2.1 ; lows, 27.2 \pm 1.7$)$ and a medial target (P3, highs, 14.8 \pm 1.9 ; lows, 15.2 \pm 1.00$)$ with respect to the starting point (Fig. 1). Active and passive movements series were performed in separate blocks. For each combination of conditions (active, passive movement; correct, incorrect visual feedback; P1, P2, P3 targets), 5 trials were averaged for a total of 60 movements performed in four experimental series of 8 minutes each, applied in counterbalanced order. The order of tasks within each series was randomly generated by a computer program which ensures exactly 5 trials for all the possible combinations.

\section{Statistical analysis}

Preliminary analyses (SPSS.15 statistical package) were performed by applying repeated measures ANOVAs to the movements velocity and to the delay between the stop signal and the movements end (stop time), according to a 2 Hypnotizability (highs, lows) х 2 movement Condition (passive, active) x 3 Target (P1,P2, P3) x 2 Visual feedback (correct, incorrect) design. The absolute error in report (absolute values of the difference between the reported and real hand positions) and under/overestimation of the hand position (constant error, relative values of the same difference) were analysed through repeated measures ANOVA according to the same design. The Geenhouse-Geisser $\varepsilon$ correction for non- sphericity was applied when necessary. Contrast analysis between targets and unpaired t test between highs and lows were used when appropriate. Level of significance was set at $\mathrm{p}<.05$.

\section{Results}

\section{Preliminary analyses}

Repeated measures ANOVA revealed that the movement mean velocity was greater during active than during passive movements for $\mathrm{P} 1($ mean $\pm \mathrm{SD}(\mathrm{cm} /$ sec); active: $53.28 \pm 17.42$; passive: $37.37 \pm 10.93$; $\mathrm{t}=9.586, \mathrm{p}<.001$ ) and $\mathrm{P} 2$ (active: $60.1 \pm 18.09$; passive: $51.26 \pm 15.04 ; \mathrm{t}=5.726, \mathrm{p}<0.0001)$, whereas no significant difference was observed for P3 (active: $63.65 \pm 19.59$; passive: 60.66 \pm 17.28 ). However, there was no significant hypnotisability-related difference in the movement velocity in both active

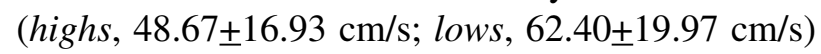


Table I. - Absolute error (mean, SD) in the hand position detection (cm).

\begin{tabular}{|c|c|c|c|c|c|c|c|c|c|}
\hline \multirow[b]{3}{*}{ visual feedback } & \multirow[b]{3}{*}{ target } & \multicolumn{4}{|c|}{ active } & \multicolumn{4}{|c|}{ passive } \\
\hline & & \multicolumn{2}{|c|}{ highs } & \multicolumn{2}{|c|}{ lows } & \multicolumn{2}{|c|}{ highs } & \multicolumn{2}{|c|}{ lows } \\
\hline & & mean & SD & mean & SD & mean & SD & mean & SD \\
\hline \multirow[t]{3}{*}{ correct } & $\mathrm{P} 1$ & 0,82 & 0,82 & 0,54 & 0,56 & 0,47 & 0,31 & 0,43 & 0,47 \\
\hline & P2 & 1,10 & 0,92 & 1,00 & 0,66 & 0,88 & 0,55 & 0,88 & 0,55 \\
\hline & P3 & 0,91 & 0,84 & 1,73 & 1,19 & 1,36 & 0,59 & 1,08 & 0,64 \\
\hline \multirow[t]{3}{*}{ incorrect } & $\mathrm{P} 1$ & 1,59 & 0,62 & 1,13 & 0,65 & 1,03 & 0,55 & 1,06 & 0,70 \\
\hline & P2 & 1,28 & 0,96 & 1,51 & 0,83 & 1,31 & 0,84 & 1,11 & 0,85 \\
\hline & P3 & 1,11 & 0,95 & 2,19 & 2,20 & 1,42 & 0,70 & 1,38 & 0,66 \\
\hline
\end{tabular}

Note. higher absolute error $(\mathrm{cm})$ corresponds to lower accuracy.

P1, P2, P3: lateral, intermediate, medial target.

and passive movements (highs, 48.39 $\pm 15.87 \mathrm{~cm} / \mathrm{s}$; lows, $51.14 \pm 21.06 \mathrm{~cm} / \mathrm{s}$ ).

Highs and lows did not differ in the time lag between the stop signal and the end of their active

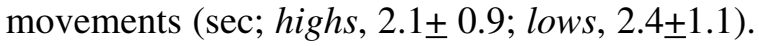

\section{Absolute Error}

A summary of the significant effects and interactions concerning the hand's localization absolute error is reported in Table I. The incorrect visual feedback increased the error of reports in all participants (Table I, II). In contrast, decomposition of the significant hypnotizability $\mathrm{x}$ target $\mathrm{x}$ movement condition interaction (Table II, Fig. 2) revealed that: a) lows exhibited the same error for active and passive movements; their lowest and highest error occur for the lateral and medial hand position, respectively; b) highs showed the same error for all hand positions for active movements, whereas their error increases from the lateral to the medial hand position for passive movements (Table II). Moreover, c) the highs' error was lower in the active than in the passive movements directed toward the medial target and greater in the passive than in the active movements directed toward the lateral target (Table II, Fig. 2). Finally, the highs' error (d) was significantly lower than the lows' one for the active movements directed toward the medial target.

Other possible effects of hypnotizability are likely to have been underestimated owing to low effect size (hypnotizability, $\eta^{2}=.009$; hypnotizability $\mathrm{x}$ visual feedback, $\eta^{2}=.004$; hypnotizability x movement condition $x$ visual feedback, $\eta^{2}=.001$ )

\section{Constant Error}

ANOVA on the constant error (relative values) revealed a significant targetxvisual feedback interaction $\left(\mathrm{F}_{(2,40)}=3.894, \mathrm{p}=.046, \eta^{2}=.197\right)$ whose decomposition showed overestimation decreasing from the medial to the lateral hand position (which was underestimated) when the visual feedback was correct, and overestimation for all hand positions in the presence of incorrect visual feedback (Fig. 3). Under/overestimation did not differ between highs and lows.

\section{Discussion}

Hypnotizability modulates the position sense. Indeed, lows exhibit similar accuracy for active and passive movements and are more accurate at the lateral target than at the intermediate and medial ones in both movement conditions. In contrast, highs perform better at the lateral target only after passive movements, and do not show target-related differences in the accuracy of the hand position detection after active movements. As a consequence, they perform better than lows at the medial target and worse than them at the lateral one. The observed hypnotisability related differences are reliable because they cannot be attributed to differences between highs and lows in the movements' velocity and, for active movements, to differences in the time lag between the stop signal and the end of movement .

We hypothesize that, in highs, the absence of significant difference in accuracy after the active movement 


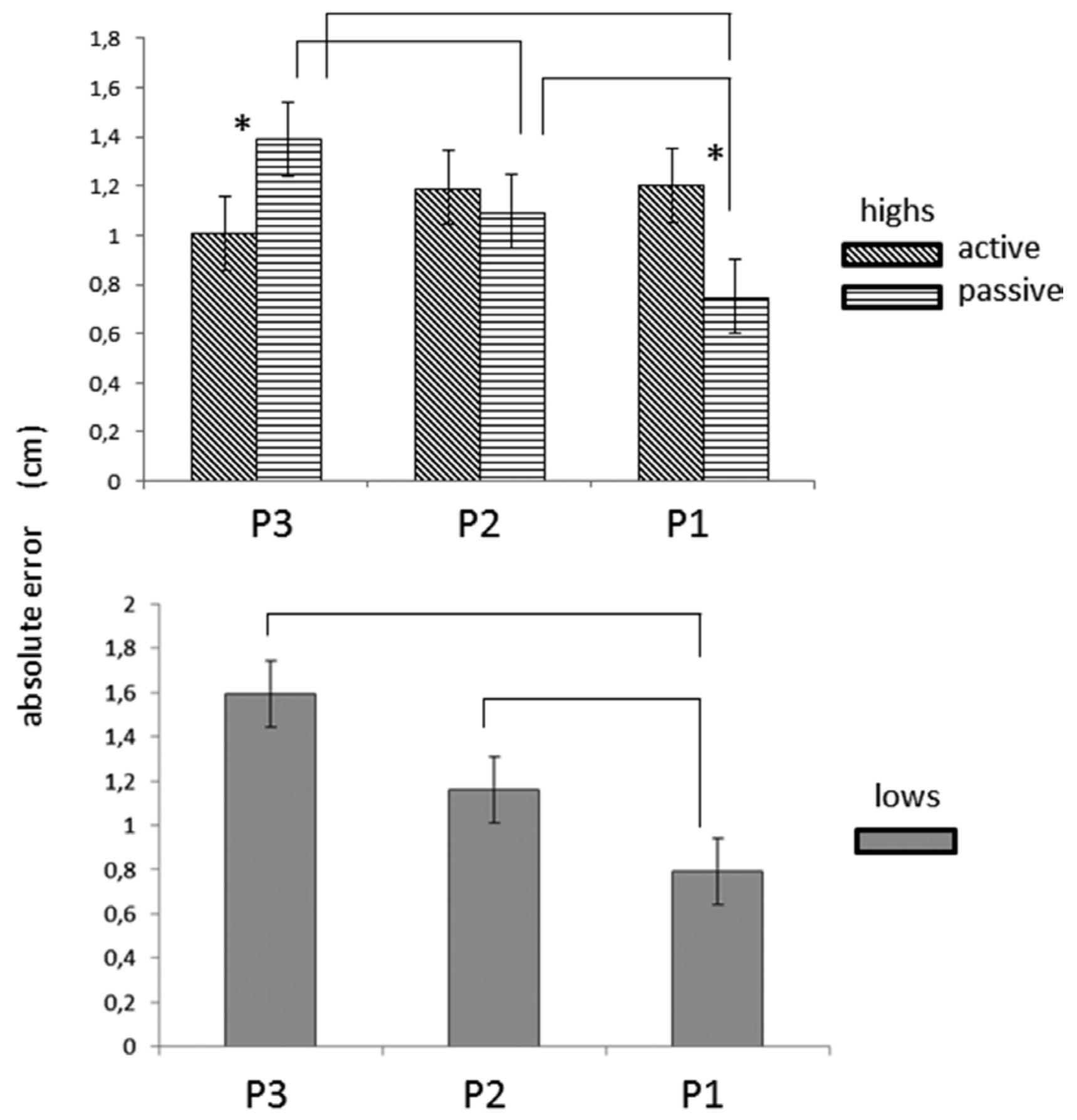

Fig. 2. - Absolute error in the detection of the hand position in highs and lows. P1, P2, P3: lateral, intermediate, medial final hand positions. ${ }^{*}$, significant difference between active and passive movements; lines, significant differences between targets.

toward the three targets is due to the efference copies of motor commands which, in these participants, can compensate the lower amount of information deriving from the movement toward the medial and intermediate target. Also the highs' greater accuracy with respect to lows in the detection of the medial target after active movements could depend on greater enhancement of their proprioceptive sensitivity by efference copies (Paillard and Brouchon 1968). It is noticeable, in fact, that the highs' cerebellum may exert a lower motor inhibition than lows' (Santarcangelo, 2014; Di Gruttola et al., 2014; Menzocchi et al, 2015), which could be 


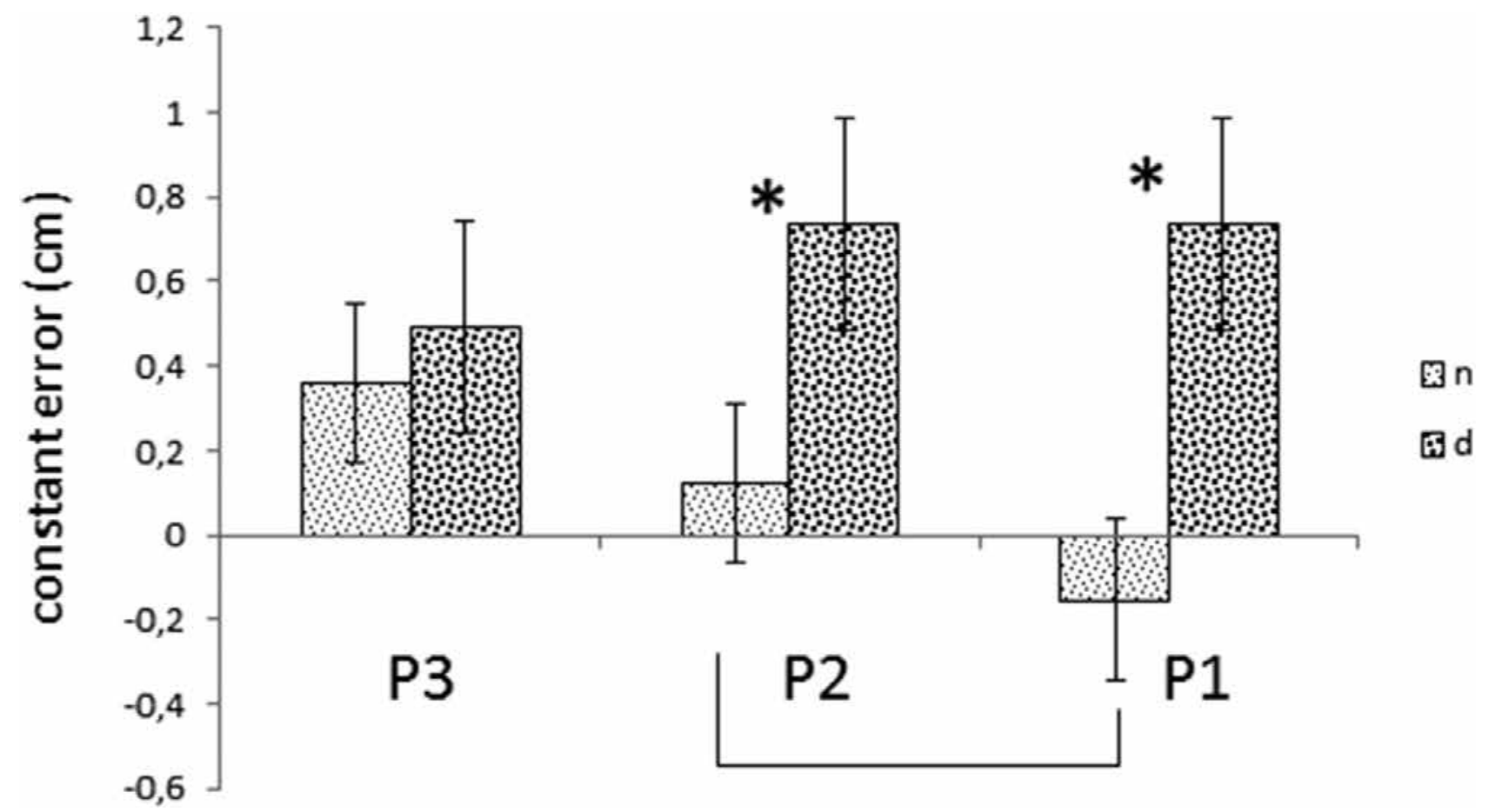

Fig. 3. - Constant error in the hand position detection. PI, P2, P3: lateral, intermediate, medial final hand positions. *, significant differences between estimation with correct or incorrect visual feedback; lines, significant difference in estimation between hand positions

responsible for greater increases of the activity of gamma motoneurons (Capaday et al., 2013; Paillard and Brouchon, 1968). The absence of significant hypnotizability-related difference in the detection of intermediate and lateral targets can be accounted for by the larger availability of peripheral information characterizing the movements toward these more distal targets and reducing the impact of efference copies.

In contrast to the possible role of the efference copies of motor commands in the enhancement of proprioceptive sensitivity, their predictive role cannot account for the difference observed between highs and lows because participants did not have any knowledge of the final point of their movement, which was imposed by the occurrence of an unpredictable beep.

The greater accuracy exhibited by lows at the lateral target for both active and passive movements and by highs in passive conditions suggests that the pre-eminent involvement joint capsules/ ligaments receptors, as joint receptors usually fire maximally at one of the extreme of the joint's range, where they work as indicators of possible damage (Clark and Burgess, 1975; Ferrell,1980).

The similar accuracy observed for active and passive movements in lows is in line with the reports concern- ing samples not selected for hypnotisability (Capaday et al., 2013) and, thus, most likely including a larger number of lows and medium hypnotizables than of highs (Balthazard and Woody 1989). The instruction to pay attention to the unpredictable sound and stop the active movement at its occurrence, however, may have represented an additional task whose cognitive demand likely reduced the reports accuracy. Finally, the unpredictable occurrence of the sound could have contributed to reduce the possible advantage of active movements in the hand position detection. In fact, another study in which the active movement was interrupted by a sound reported similar accuracy in the identification of the position of the passively and actively placed right hand (Jones et al., 2010).

The incorrect visual feedback affects the hand localization in highs and lows to the same degree, although this finding may have been biased by the low effect size of the hypnotisability $\mathrm{x}$ visual feedback interaction. It was effective in both movement conditions and for all targets, in line with the great importance of visual information generally observed in sensorimotor integration tasks (Judkins and Scheidt, 2014; Ladwig et al., 2013). However, the absence of difference between highs and lows (to be replicated in larger samples) may 


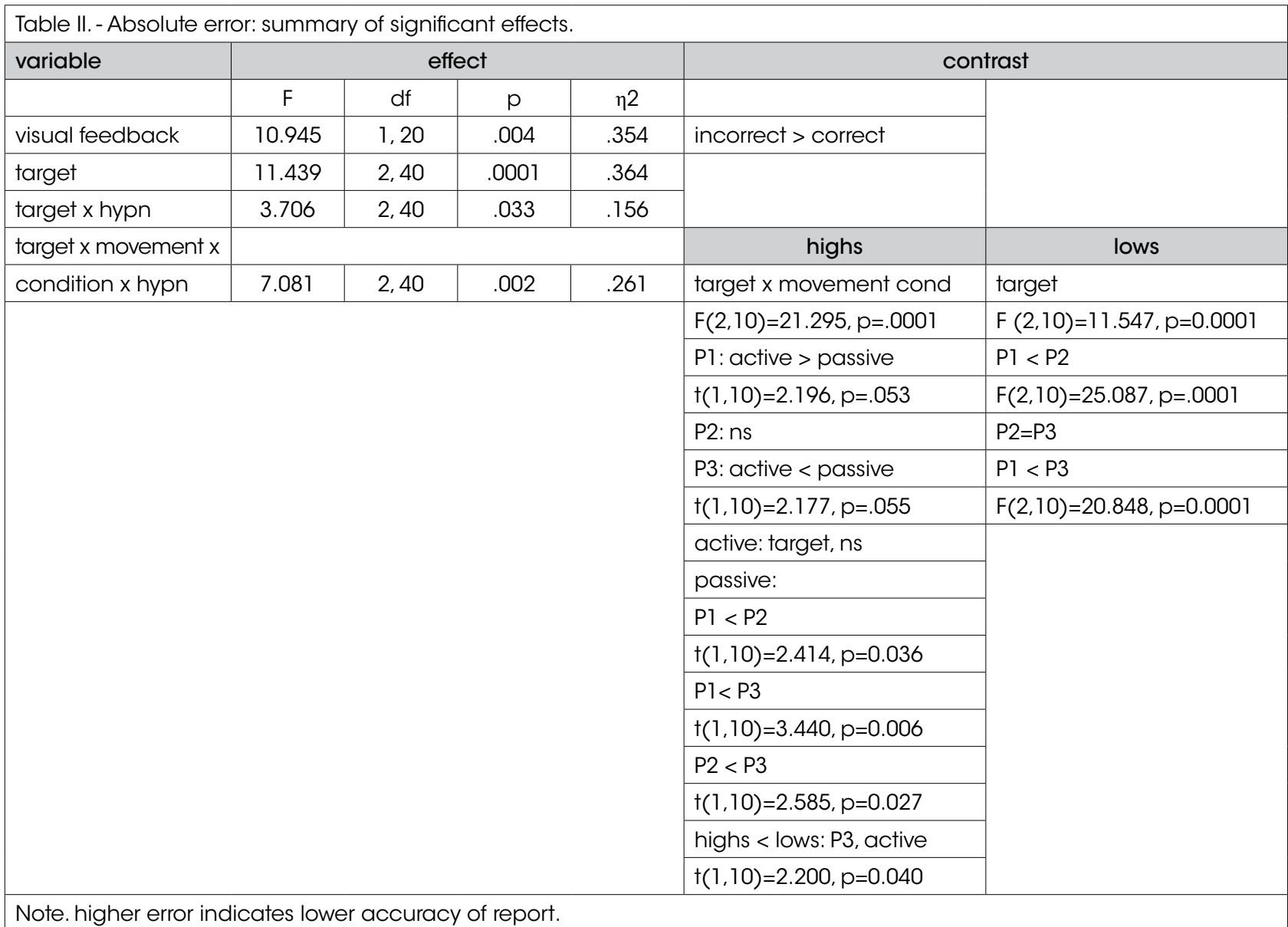

indicate that, despite the highs' peculiar characteristics of absorption (Tellegen and Atkinson, 1974), which theoretically makes their performance more vulnerable to the incorrect visual feedback of the hand position, visual interference was not sufficient to reduce the highs' advantage in the active proprioception with respect to lows. Moreover, since highs did not perform better than lows in the presence of correct visual feedback, we may argue that their advantage was entirely due to kinaesthetic information.

The tendency to overestimation of the final hand position is in line with other authors' reports (Cameron et al., 2014). The occurrence of significantly different overestimation between the effect of the correct or incorrect visual feedback for the larger movements (targeting P1 and P2), but not for the most medial hand position (P3), can be attributed to the longer time required by visual stimuli with respect to the proprioceptive information to reach brain areas (Fetz et al., 1980; Soso and Fetz, 1980; Evarts and Fromm, 1981). This difference may account for the effect of the visual feedback only for longer movement times.

Important limitations of the study are the low number of participants compared to the number of conditions and the absence of males. The present study should be considered exploratory and findings should be replicated in more numerous samples including both gender, as gender may influence the position sense (Dunn et al., 2015). In addition, the hand position was detected by relying only on the shoulder proprioception. The shoulder was a good model for our aims, as acuity is greater for proximal than for distal joints (Goldscheider, 1889), but results cannot be generalized to other joints.

In conclusion, our findings support the view that high and low hypnotizable individuals have different acuity of the position sense and that central commands seem to be more relevant in the highs' than in the lows' sensori-motor integration. Findings encourage further investigation potentially useful in the set- up of individualized neuro-rehabilitative protocols. 


\section{References}

Adamovich S.V., Berkinblit M.B., Fookson O., Poizner H. Pointing in 3D Space to Remembered Targets. I. Kinesthetic versus Visual Target Presentation. J. Neurophysiol., 79: 2833-46, 1998.

Balthazard C.G., Woody E.Z. Bimodality, Dimensionality, and the Notion of Hypnotic Types. Int.J.Clin. Exp. Hypn., 37:70-89, 1989.

Bergamasco M., Avizzano C.A., Frisoli A., Ruffaldi E., Marcheschi S. Design and Validation of a Complete Haptic System for Manipulative Tasks. Advanced Robotics., 20: 367-89, 2006.

Cameron B.D., de la Malla C., López-Moliner J.L. The Role of Differential Delays in Integrating Transient Visual and Proprioceptive Information. Front. Psychol., 5: 50, 2014.

Capaday C., Darling W.G., Stanek K., Van Vreeswijk C. Pointing to Oneself: Active versus Passive Proprioception Revisited and Implications for Internal Models of Motor System Function. Exp. Brain Res,229: 171-80, 2013.

Carli G., Cavallaro F.I., Rendo C., Santarcangelo E.L. Imagery of Different Sensory Modalities:Hypnotizability and Body Sway. Exp. Brain Res.,179: 147-54, 2007.

Carli G., Cavallaro F.I., Santarcangelo E.L. Hypnotisability and Imagery Modality Preference: Do Highs and Lows Live in the Same World? Contemp. Hyp., 24: 64-75, 2007.

Carli G., Manzoni D., Santarcangelo E.L. Hypnotizability-Related Integration of Perception and Action. Cogn. Neuropsychol., 25: 1065-76, 2008.

Castellani E., Carli G., Santarcangelo E.L. Visual Identification of Haptically Explored Objects in High and Low Hypnotizable Subjects. Int.J. Clin. Exp. Hypn, 59: 250-65, 2011.

Clark F. J., Burgess P.R. Slowly Adapting Receptors in Cat Knee Joint: Can They Signal Joint Angle? J. Neurophysiol., 38: 1448-63, 1975.

Craske B., Crawshaw M. Shifts in Kinesthesis through Time and after Active and Passive Movement. Percept. Motor Skills, 40: 755-61, 1975.

Cui F., Arnstein D., Thomas R.M., Maurits N.M., Keysers C., Gazzola V. Functional Magnetic Resonance Imaging Connectivity Analyses Reveal Efference-Copy to Primary Somatosensory Area, BA2. PloS One, 91: e84367, 2014.

De Pascalis V., Bellusci A., Russo P.M . Italian Norms for the Stanford Hypnotic Susceptibility Scale, Form C. Int .J.Clin .Exp. Hypn., 48: 315-23, 2000.
Di Gruttola F., Orsini P., Carboncini M.C., Rossi B.,Santarcangelo E.L. Revisiting the Association between Hypnotisability and Blink Rate. Exp. Brain Res., 232: 3763-69, 2014.

Dunn W., Griffith J.W., Sabata D., Morrison M.T., MacDermid J.C., Darragh A., Schaaf R., Dudgeon B., Connor L.T., Carey L., Tanquary J. Measuring change in somatosensation across the lifespan. Am. J. Occup. Ther., 69: 1-9, 2015.

Evarts E. V., Fromm C. Transcortical Reflexes and Servo Control of Movement. Can .J. Physiol. Pharmacol.l, 59: 757-75, 1981.

Ferrell W. R. The Adequacy of Stretch Receptors in the Cat Knee Joint for Signalling Joint Angle throughout a Full Range of Movement. J. Physio.l, 299: 85-99, 1980.

Fetz E., Finocchio D.V., Baker M.A., Soso M.J. Sensory and Motor Responses of Precentral Cortex Cells during Comparable Passive and Active Joint Movements. J. Neurophysiol., 43(4):1070-89, 1980.

Fuentes C.T., Bastian A.J. Where Is Your Arm? Variations in Proprioception across Space and Tasks. J. Neurophysiol., 103: 164-71, 2010.

Goldscheider A. Untersuchungen Uber Den Muskelsinn. In Archives Für Anatomie Und Physiologie, 3: 369-502, Liepzig, 1889.

Green J.P., Barabasz A.F., Barrett D., Montgomery G.H. Forging Ahead: The 2003 APA Division 30 Definition of Hypnosis. Int. J. Clin. Exp. Hypn., 53: 259-64, 2005.

Gritsenko V., Krouchev N.I., Kalaska J.F. Afferent Input, Efference Copy, Signal Noise, and Biases in Perception of Joint Angle during Active versus Passive Elbow Movements. J. Neurophysiol., 98: 1140-54, 2007.

Huber A. The Role of Motivational Factors in the Relationship between Hypnotizability and PainInducing Imagery. Neurosci. Lett., 557: 181-83, 2013.

Jones S.A.H., Cressman E.K., Henriques D.Y. Proprioceptive Localization of the Left and Right Hands. Exp Brain Res., 204: 373-83, 2010.

Judkins T., Scheidt R.A. Visuo-Proprioceptive Interactions during Adaptation of the Human Reach. J. Neurophysiol., 111: 868-87, 2014.

Ladwig S., Sutter C., Musseler J. Intra- and Intermodal Integration of Discrepant Visual and Proprioceptive Action Effects. Exp. Brain Res., 231: 457-68, 2013.

Laufer Y., Hocherman S., Dickstein R. Accuracy of Reproducing Hand Position When Using Active 
Compared with Passive Movement. Physiother. Res. Int., 6, 2001.

Leube D.T., Knoblich G., Erb M., Grodd W., Bartels M., Kircher T.T.J. The Neural Correlates of Perceiving One's Own Movements. NeuroImage, 20: 2084-2090, 2003.

Medina J., Jax S.A., Brown M., Coslett H.B. Contributions of Efference Copy to Limb Localization: Evidence from Deafferentation. Brain Res., 1355: 104-111.

Menzocchi M., Mecacci G., Zeppi A., Carli G., Santarcangelo E.L. Hypnotizability and performance on a Prism Adaptation Test. Cerebellum, [Epub ahead of print], 2015

Padilla-Castañeda M.A., Frisoli A., Pabon S., Bergamasco M. The Modulation of Ownership and Agency in the Virtual Hand Illusion under Visuotactile and Visuomotor Sensory Feedback. Presence: Teleoperators and Virtual Environments, 23: 209-225, 2014.

Paillard J., Brouchon M. Active and Passive Movements in the Calibration of Position Sense. In: The Neuropsychology of Spatially Oriented Behavior, Freedman S, 37-55. Dorsey, Homewood, 1968.
Santarcangelo E.L. New Views of Hypnotizability. Front.Behav. Neurosci.,8: 224, 2014

Santarcangelo E. L., Cavallaro E., Mazzoleni S., Marano E., Ghelarducci B., Dario P., Micera S., Sebastiani L. Kinematic Strategies for Lowering of Upper Limbs during Suggestions of Heaviness: A Real-Simulator Design. Exp .Brain Res., 162: 35-45. 2005.

Santarcangelo E. L., Scattina E., Carli C., Ghelarducci B., Orsini P., Manzoni D. Can Imagery become Reality? Exp. Brain Res., 206: 329-335, 2010.

Santarcangelo E.L., Scattina E., Carli G., Macerata A., Manzoni D. Hypnotizability-Dependent Modulation of Postural Control: Effects of Alteration of the Visual and Leg Proprioceptive Inputs. Exp. Brain Res., 191: 331-340, 2008.

Soso M. J., Fetz E. Responses of Identified Cells in Postcentral Cortex of Awake Monkeys during Comparable Active and Passive Joint Movements. J. Neurophysiol., 43: 1090-1110, 1980.

Tellegen A., Atkinson G. Openness to Absorbing and Self-Altering Experiences ('absorption'), a Trait Related to Hypnotic Susceptibility. J. Abn. Psychol., 83: 268-277, 1974. 